\title{
Introducing a quality improvement programme to primary healthcare teams
}

\author{
Hilary Hearnshaw, Stuart Reddish, Denise Carlyle, Richard Baker, Noelle Robertson
}

\begin{abstract}
Objectives-To evaluate a programme in which quality improvement was facilitated, based on principles of total quality management, in primary healthcare teams, and to determine its feasibility, acceptability, effectiveness, and the duration of its effect.

Method-Primary healthcare teams in Leicestershire $(n=147)$ were invited to take part in the facilitated programme. The programme comprised seven team meetings, led by a researcher, plus up to two facilitated meetings of quality improvement subgroups, appointed by each team to consider specific quality issues.

Outcome measures-To assess the effect and feasibility of the programme on improving the quality of care provided, the individual quality improvement projects undertaken by the teams were documented and opportunities for improvement were noted at each session by the facilitator. The programme's acceptability was assessed with questionnaires issued in the final session to each participant. To assess the long term impact on teams, interviews with team members were conducted 3 years after the programme ended.
\end{abstract}

Results-10 of the 27 teams that initially expressed interest in the programme agreed to take part, and six started the programme. Of these, five completed their quality improvement projects and used several different quality tools, and three completed all seven sessions of the programme. The programme was assessed as appropriate and acceptable by the participants. Three years later, the changes made during the programme were still in place in three of the six teams. Four teams had decided to undertake the local quality monitoring programme, resourced and supported by the Health Authority.

Conclusions-The facilitated programme was feasible, acceptable, and effective for a few primary healthcare teams. The outcomes of the programme can be sustained. Research is needed on the characteristics of teams likely to be successful in the introduction and maintenance of quality improvement programmes.

(Quality in Health Care 1998;7:200-208)

Keywords: primary healthcare teams; quality management; quality improvement; facilitation
Introduction

In the United Kingdom, national policy for the development of audit in primary health care has evolved towards the creation of a wider quality assurance programme with every provider unit having a system of quality assurance. ${ }^{1}$ One means of fulfilling this objective is offered by the quality management approaches successfully adopted in industrial settings - such as total quality management ${ }^{2}$ and continuous quality improvement. ${ }^{34}$ Total quality management has been described as a participative, systematic approach to planning and implementing a continuous organisational improvement process. ${ }^{5}$ It provides a systematic approach to quality and recognises that only through the management of people working in teams, groups, and organisations can the quality of services be improved. ${ }^{6}$ Such a philosophy of a patient focused approach to quality improvement for primary health care requires a strategic approach to quality management supported by all the members of the team, not least the general practitioners. The implementation of these elements requires three stages. ${ }^{5}$ Firstly, an awareness develops that quality issues must be considered. Secondly, a quality improvement programme is identified as the means of attaining the quality objectives. Thirdly, quality improvement is implemented by all members of the team.

The successful introduction of quality improvement into a primary healthcare team can be influenced by the characteristics of the team. Characteristics which have been shown to have beneficial influence include willingness to consider and accept innovation, tolerance of diversity, commitment to the success of the team and collaboration between team members, ${ }^{7}$ the balance of roles of team members, ${ }^{8}$ resources of time and people available for the task, and the positive underlying beliefs and attitudes of team members towards the task. ${ }^{9}$ The concept of team culture, comprising such characteristics, has been depicted by Hardy as "the way things are done here". ${ }^{10}$ It is likely that a quality improvement programme will succeed only in those primary healthcare teams which have at least some of these characteristics.

By contrast with the characteristics which may lead to success, some of the potential obstacles to the introduction of total quality management in industrial settings have also been identified. ${ }^{11}{ }^{12}$ These include insufficient resources to start or maintain the programme, limited objectives which define the scope for quality management in a restricted way, lack of inspirational leadership, lack of commitment from leaders, and lack of rewards for success. 
Table 1 Elements of total quality management ${ }^{17}$ included in the foundations for quality improvement programme

\begin{tabular}{ll}
\hline TQM element & Elements in the foundations for quality improvement programme \\
\hline Leadership & All team members involved, commitment from GPs, consensus decision making, and implementation \\
Culture & Assessment of the team culture by the team members \\
Customer focus & Collecting patients' views of the services provided \\
Strategic planning for quality & Identification of the team's quality improvement objectives \\
Information and analysis & Learning methods to achieve those objectives, building on any existing knowledge of clinical audit \\
Information and analysis & Using a systematic analysis to understand the quality problems and identify obstacles \\
Change & Using methods to overcome those obstacles and achieve the objectives \\
Culture & Using teamwork to enhance the process \\
\hline
\end{tabular}

The successful introduction of a quality improvement programme in primary health care may thus depend on teams with the desirable characteristics being encouraged to identify and consider such obstacles.

The number of successful examples of total quality management in primary healthcare settings has been small. ${ }^{13}$ In a study in the Oxford region, 18 practices took part in a total quality programme and 16 succeeded in implementing quality improvement methods. ${ }^{14}$ This programme was delivered through an intensive 2 day residential course and three 1 day seminars for three members of each practice team, who then had the task of implementing total quality with support from a facilitator. It has previously been shown ${ }^{15}$ that facilitation can be effective in stimulating the development of a team approach to audit. Studies of the long term effects of facilitation are rare, but one has suggested that the effect of a facilitator may only last for the period of intervention. ${ }^{16} \mathrm{How}-$ ever, ongoing facilitation for primary healthcare teams can be provided by audit support staff of primary care audit groups.

A programme was designed for the facilitated implementation of quality improvement in primary healthcare teams. It included the three stages of implementation already described, ${ }^{5}$ the identification of the characteristics of a team, and the identification and rectification of potential obstacles for the team. A study was undertaken to evaluate the feasibility, effectiveness, and acceptability of the programme and the longevity of its effect, both in persistance of improvements made during the project and persistance of changes of attitude to quality.

\section{Method}

QUALITY IMPROVEMENT PROGRAMME

Recruitment

A leaflet outlining the programme (called foundations for quality improvement) and a letter of invitation was sent to all 147 general practices in Leicestershire in March 1994. The leaflet stated that "This programme will not only help the team clarify its objectives but also provide instruction and guidance on how to achieve them and go on achieving them". The programme offered "...an introduction to the strengths and benefits of quality management in general practice". Personal visits were made by a researcher (DC) to those teams who asked for further details to give them information about the project programme and the level of commitment expected. Those who agreed to take part followed the programme which was facilitated by a research team member (DC or
SR) and the local medical audit advisory group support staff who had previous experience in facilitating primary healthcare teams with clinical audit, but limited experience of other quality improvement methods. A second member of the research team attended the first presentation of each session to observe the process and to provide feedback for the facilitator. The teams were offered financial support should staff be required to attend outside their normal hours and the doctors were offered postgraduate education allowance certificates for their participation.

Aims and objectives of the programme

The programme was designed to leave each team with the foundations of the management of quality improvement in place and ready to continue their quality management after the programme was completed. This programme did not attempt to introduce a total quality management philosophy to practices, ${ }^{261217}$ which would require the application of quality management procedures to all aspects of the organisation: policy setting; strategy; structure; and culture. ${ }^{17}$ Rather, it included elements of total quality management, applied in a limited way to introduce quality improvement through small steps, relevant to primary health care (table 1).

By the end of the programme, members of each team would ( $a$ ) be aware of their own and their colleagues' approaches to quality and teamwork, $(b)$ have identified the obstacles they need to overcome for their quality management to succeed, (c) have experienced successful team consensus decision making, and $(d)$ have experienced successful completion of a first quality improvement project, which included measurement and change.

\section{Programme content}

The content and presentation of the programme were based on the process of experiential learning in which the concepts of quality management would first be acquired and then put into practice, guided by the facilitator. ${ }^{18}$ Seven meetings of 1.5-2 hours in duration were held at about monthly intervals with each team, at the practice, following the format evaluated in a previous study ${ }^{15}$ and led by the facilitator. The content of the programme and the expected achievement of its objectives are outlined in box 1 .

\section{Understanding team culture}

The questionnaire used in session 1 to help teams identify their culture ${ }^{19}$ is given in box 2 . The results were discussed in session 3, enabling each team to identify aspects of its 
Session 1

- Assessment of the present situation with a questionnaire on the team's organisational culture ${ }^{19}$

- Introduction to quality improvement with a short video ${ }^{22}$

- Identification of barriers to the introduction of quality improvement by brainstorming

Session 2:

- Experience consensus decision making and synergy: a survival situation exercise $^{23}$ was used to provide experience in reaching consensus decisions and show the synergy of team work, in which the outcome of a team decision can be more effective than any person's decision

- Reflection on how the groups had reached their decisions and the differences from their usual method

Session 3:

- Feedback on present team culture from the questionnaire in session 1

- Identification of strengths and weaknesses to enhance understanding of culture

- Identification of quality issues important to patients by conducting a survey of patients with the surgery satisfaction questionnaire $^{24}$ before the next session Objectives achieved so far:

- Awareness of their own and their colleagues' approach to quality and teamwork

- Obstacles to quality management identified

- Experience of successful consensus decision making

Session 4:

- Presentation of the results from the patient survey
- Use of problem solving techniques - part 1: the audit cycle

Session 5:

- Use of problem solving techniques-part 2: cause and effect diagrams, brainstorming, and flow charting

- Setting an agenda of issues to be considered and methods to be used. Prioritising problems and appointment of quality improvement groups, made up of team members, to start the agenda

Session 5A: quality improvement group session:

- Use of problem solving techniques, planning, and conducting data collection, identification of possible solutions: emphasis on identifying problems in processes of practice activities rather than in blaming individual people

- Preparation of feedback session to whole team

Session 6:

- Feedback from quality improvement group with recommendations for action-such as further audit, further analysis of a situation, or specific changes

- Consensus decision making on the action to be taken, delegation of responsibilities, and deadlines for those actions

Session 7:

- Firm plans for the next steps

- Requests for further information

- Reflection on the project

- Repeat of questionnaire on attitudes to audit and an evaluation of the programme

- Each participant who returned the questionnaires was sent a certificate of attendance

Remaining objective achieved:

- Successful completion of a quality improvement project

Box 1 Contents of the foundations for quality improvement programme used in the study.

current culture which the team might choose to change. The questionnaire was scored on the aspects of innovation seeking, planning, control and loyalty, co-operation, communication and information, and valuing staff. This questionnaire can also be of value in helping teams to identify their readiness to change.

\section{Evaluation of the programme}

The programme was evaluated by several measures. To assess the effect of the programme on improving the quality of care provided, the individual quality improvement projects undertaken by the teams were documented by recording the methods used to identify the problem and possible solutions, the data collections made, and the changes agreed upon by the teams. A quality improvement project was deemed to have been successful if the group appointed to undertake it had completed the tasks assigned to them, the whole team had agreed any changes to be made, and had made explicit plans to implement them. The tasks included identifying and collecting the appropriate data and informing the whole team, so that the source of any problems could be clearly located before remedial measures were suggested.

To examine and assess the process of the facilitator's experience, the feasibility of the programme, and to note opportunities for improvement, a record was kept of each session by the facilitator. This included noting the objectives of the session, tools and methods used, what the team had achieved and agreed, significant interactions between members of the team, any comments made about the programme by team members, and the willingness with which participants took part.

The acceptability of the programme was assessed with evaluation questionnaires issued in the final session to each participant. A response was invited, on a 5 point scale from 1 (poor), 3 (neutral), to 5 (excellent), for the appropriateness of the programme content, the helpfulness of the facilitator, the handouts, and the questionnaires, the pace and clarity of the presentations and the interest and enthusiasm 
Innovation seeking:

It is vital for business success to keep up with new developments

Try to avoid doing things in the same, predictable ways

Successful organisations generally keep one step ahead of the rest

People should always look for new ways of solving problems

Planning:

Always explore the alternatives before acting

It is essential to think ahead

A successful practice always knows where it is going

Control and loyalty:

Successful people are those who are loyal to their boss

You need to be firm and decisive to survive

Subordinates should be hard working and loyal

Control is all important

People in authority have more clout

You have to be hard and tough to get on

Cooperation:

Success comes to those who get on with others

Always try to get on with your colleagues

Working together is important

Always look for constructive ways of overcoming problems

Teamwork comes first

Communication and information:

Ideas should flow freely

Management should take care over what they say to subordinates

Open communication is best

Everyone in the practice should be kept informed

Policy decisions should always be based on sound information

Valuing staff:

Be spontaneous

Always try to improve your understanding of your job

Employees should be helped to realise their full potential

Staff should be encouraged to be enthusiastic

Box 2 Statements from the questionnaire used in session 1 to identify the practice culture. ${ }^{19}$ Each statement was scored on a seven point scale for importance of the belief in the practice, from $7=$ to a great extent to $1=$ not at all.

shown by the facilitator. The responses were analysed with Epi Info software to calculate the mean score over all responders for each question.

\section{Long term effects}

Each practice was contacted 3 years after the programme. The same researcher (SR), who had been a facilitator for the programme, interviewed the practice manager to ask whether the quality improvement programme was remembered, what had happened since the programme to the aspect of care which the quality project considered, what were the qual-
Table 2 Summary of details of practices participating in the foundations for quality improvement programme

\begin{tabular}{|c|c|c|c|c|c|}
\hline Practice & e Partners & $\begin{array}{l}\text { List size } \\
\text { to nearest } \\
1000\end{array}$ & $\begin{array}{l}\text { farman score } \\
\text { to nearest } \\
\text { whole number }\end{array}$ & Fundholding & $g$ Training \\
\hline 1 & 4 & 7000 & 21 & No & No \\
\hline 2 & 3 & 7000 & -2 & Yes & Yes \\
\hline 3 & 3 & 6000 & 31 & No & No \\
\hline 4 & 7 & 13000 & -9 & No & Yes \\
\hline 5 & 5 & 8000 & -7 & No & No \\
\hline 6 & 7 & 10000 & 23 & No & No \\
\hline
\end{tabular}

The Jarman score ${ }^{25}$ for the electoral ward in which the practice is situated indicates relatively more social deprivation the more positive the score.

ity improvement activities current in the team, and whether the programme had had any lasting effects on the practice. The interviews were recorded on tape and transcribed.

\section{Results}

\section{RECRUITMENT}

Of the 147 practices offered the programme, $27(18 \%)$ asked for more information and of these $10(7 \%)$ agreed to take part. Seventeen practices decided not to take part. Four practices were involved in renovation or moving of premises. One practice declined to take part if locum reimbursement for attendance by general practitioners was not offered. One practice manager had previous experience of total quality management in another organisation and thought that all staff would not be fully committed. One practice team thought that they were too large to meet together. In four practices none of the doctors were willing to take part although other staff wanted to. In two practices the general practitioner wanted to take part but the other staff did not. In two practices unexpected resignation of staff left the general practitioners thinking that this was not an appropriate time for them to do the programme. In two practices the practice manager was on sick leave and unable to start the programme.

Four of the 10 practice teams dropped out of the programme after agreeing to start, in one practice because of moving premises, in the second due to illness of the practice manager, in the third due to the lengthy holiday of a senior staff member, and in the fourth because the administrative staff chose not to attend the first session. Therefore, six practice teams started the programme. Five teams undertook a quality improvement project, but only three completed the full seven sessions of the programme. Details of the participating practices are shown in table 2 and their completion of the programme in table 3. Scores for each team on the team culture questionnaire are given in table 4 .

TEAM CULTURES

The characteristics of teams were shown by the culture questionnaire. Team 1 scored above the mean on four of the six aspects, and sustained the changes made during the programme. This team had a high Jarman deprivation score, and was not fundholding or a training practice. Team 2 also scored highly on all aspects and completed the programme, sustained the 
changes implemented, and was continuing with quality improvement activities. It also had a low Jarman deprivation score, was the only fundholding practice in the study, and was a training practice. There seems to be no relation between completion of this programme and whether the practice is fundholding, training, or in a deprived area. Team 3 scored below the mean of all teams on all aspects except valuing staff. This team dropped out of the programme after one doctor had acted without consulting others in the team. However, the members of the team had valued working together in the programme.

Team 4 was more than twice as large as any other team in the study. Their highest relative score was on control and loyalty. The team found it needed to learn to value all views, reflecting the existing culture of hierarchy. Having rectified that problem the team was able to continue without continued external facilitation, reflecting the internal loyalty of the team.

Team 5 scored higher than other teams on the aspect of control and loyalty. This team had one member who wished to preserve the "natural hierarchy", and their quality project showed that each doctor had independently used a different system for telephone calls. This may show the reinforcement of power hierarchies within this team. The team had low scores on innovation seeking, cooperation, and valuing staff. This was reflected in the negative attitudes initially found by the facilitator.

Team 6 had particularly low scores for control and loyalty. This was reflected in low commitment to each other and to agreed responsibilities. They also had low scores on other aspects except cooperation, in which their score was above the mean. This team did not complete their quality project and there were feelings of dissatisfaction at the end of the programme. They had not coordinated their activities as a team and had varied widely in their responses to the culture questionnaire.

The highlights of each team's progress through the programme and reports from the follow up interviews are described below.

TEAM 1

Obstacles

The brainstorming of barriers to quality improvement in the first session was difficult for this team as they did not seem comfortable in admitting difficulties. However, at the second session team members reported becoming aware of interaction behaviour - such as praising colleagues. The survival exercise was one of the most popular activities used in the programme. It was used to provide experience of consensus decision making, in subgroups of the team, by asking participants to imagine that they were cast in a life threatening situation in which important choices must be made. In this team it gave a clear example of how one person's contribution to a group decision was ignored. One part time secretary, of all the people in all the groups, had a better individual score than the group score. Although she knew the right answers she was not listened to by other members of her group in reaching their group decisions. The feedback at the end of the exercise highlighted what had been happening. Thus two obstacles were identified as not admitting difficulties and not listening to all contributions.

The examination of strengths and weaknesses of the team in session 3 was undertaken enthusiastically in a non-threatening atmosphere. Humour indicated good working relations and there was easy agreement in choosing a topic to consider in session five. This suggested that the obstacles had been reduced.

\section{Quality improvement project}

The quality project group considered the problem of incoming telephone calls with a cause and effect diagram. They identified that the problem was too much time spent on the telephone by receptionists and the cause was requests for repeat prescriptions. The group presented to the rest of the team a flow chart of the process for improvement with options for action. The team agreed that the quality improvement group should organise the collection of data on the number and duration of calls and the reason for each call. Results showed that $20 \%$ of calls were for prescriptions which was less than expected, but that they took more time than other calls. After discussion the team agreed a set of practical solutions to change the process for repeat prescriptions and agreed a timetable for implementation and reassessment.

\section{Reflection}

The team expressed a sense of achievement in completing the programme and made plans for the next issue to be tackled.

Table 3 Activities and levels of completion of teams in the foundations for quality improvement programme

\begin{tabular}{|c|c|c|c|c|c|c|c|}
\hline \multirow[b]{2}{*}{ Team } & \multirow[b]{2}{*}{ Topic agreed } & \multirow[b]{2}{*}{$\begin{array}{l}\text { Method used by the } \\
\text { quality improvement } \\
\text { group }\end{array}$} & \multirow[b]{2}{*}{ Outcome of project } & \multirow[b]{2}{*}{$\begin{array}{l}\text { Quality } \\
\text { improvement } \\
\text { group session } 5 A\end{array}$} & \multirow[b]{2}{*}{$\begin{array}{l}\text { Whole team } \\
\text { sessions } \\
\text { completed (n) }\end{array}$} & \multicolumn{2}{|l|}{ Three years later } \\
\hline & & & & & & Project change & $\begin{array}{l}\text { Quality } \\
\text { activity }\end{array}$ \\
\hline 1 & Incoming telephone calls & $\begin{array}{l}\text { Cause and effect then } \\
\text { data collection }\end{array}$ & Agreed action implemented & $\mathrm{Y}$ & 7 & Change sustained & Some \\
\hline 2 & Incoming telephone calls & $\begin{array}{l}\text { Data collection then } \\
\text { brainstorming }\end{array}$ & $\begin{array}{l}\text { Agreed action implemented. } \\
\text { Second data collection } \\
\text { planned }\end{array}$ & $\mathrm{Y}$ & 7 & Change sustained & Ongoing \\
\hline 3 & None & None & None & $\mathrm{N}$ & 5 & No change recorded & $\begin{array}{l}\text { Not } \\
\text { known }\end{array}$ \\
\hline 4 & Internal communications & Discussion & Agreed action implemented & $\begin{array}{l}\text { Y (without } \\
\text { facilitator) }\end{array}$ & 5 & Change sustained & Some \\
\hline 5 & Incoming telephone calls & Cause and effect & Agreed action implemented & $\mathrm{Y}$ & 7 & Change sustained & None \\
\hline 6 & Repeat prescriptions & Flow chart & Discouragement & $\mathrm{Y}$ & 5 & No change recorded & None \\
\hline
\end{tabular}


3 Years later

The programme and the project on telephone calls had been remembered clearly. The practice did not take repeat prescriptions by telephone, but there were still problems with the repeat prescriptions system and the telephones were still always busy. An audit of repeat prescribing was ongoing. Full team meetings, started as a result of the team culture discussions, had continued every 8 weeks since the programme and informal staff meetings took place over lunch. The system for identifying problems was still effective, but ways to tackle them were not being found. External support would have been welcomed to get things moving again.

TEAM 2

Obstacles

The culture questionnaire was said by one doctor to measure only perceptions by team members of how the team worked and not how it "really" works. This reluctance to accept others' opinions as genuine was one obstacle. A second obstacle was that the attendance by doctors was sporadic. Neither of these was successfully rectified during the programme, but the completion of a quality project was achieved by other team members despite this. This team had different team members present at each session leading to confusion and reduced commitment from the regular attenders. The team comprised staff from two practice premises who seemed to have different needs, different objectives, and initially found it difficult to interact. Discussion showed that staff who had no previous training or instruction on audit were feeling out of their depth. Session 4 on audit encouraged their involvement and seemed to overcome that obstacle.

\section{Quality project}

An enthusiastic quality improvement group was appointed to consider the topic of incoming telephone calls. They collected data and identified that morning calls asking for information on surgery times and repeat prescriptions would be better handled at other times of the day. Brainstorming was used by the group to prepare a short list of possible methods for altering patient behaviour to encourage calls to be made at other times. This was presented to the team, actions were agreed, and collection of follow up data was planned. It was agreed that the quality improvement group should continue to tackle other items on the agenda, but that membership of the group should be deter- mined by the topic and thus spread the responsibility and opportunity for participation in what was seen to be an important and influential activity.

\section{Reflection}

Reflection on the programme by the team during the last session showed that the lack of full commitment from the doctors had affected the commitment of everyone in the team. The staff thought that the quality improvement groups were worth pursuing if only as a means to get the doctors' attention drawn to the problems.

\section{Years later}

The improvement in managing incoming telephone calls had continued for nearly 3 years until a new telephone system was installed which allowed direct calls. A folder of written standards and protocols had been created and implemented with full support from the doctors. The team had monitored and evaluated their adherence to their own protocols. The practice was still enthusiastic and active in quality improvement and had completed the local "blueprint" programme, ${ }^{20}$ an adaptation of the King's Fund organisational audit ${ }^{21}$ which implemented monitoring of performance against standards in certain topic areas. The practice manager commented that having an external facilitator had been more effective in taking things forward than a member of the team could have been.

\section{TEAM 3}

Obstacles

This team functioned in a hierarchical style which meant that some team members initially found it difficult to join in the brainstorming of barriers to quality improvement in session 1 . Between the first and second session they had independently generated a list of possible solutions to their barriers. The team had already discussed some of the issues raised, at a practice meeting, and steps had been taken to improve information and expectations about waiting times. The survival exercise was enjoyed with much humour, successful consensus decisions were reached by team discussion, and synergy was achieved. The brainstorming of strengths and weaknesses of the team in session 3 produced much more participation than the brainstorming in session 1.

The discussion of the results of the culture questionnaire in the third session exposed the power of those at the top of the hierarchy and the lack of autonomy felt by those lower down.

Table 4 Mean scores from the questionnaire used in session 1 to identify the practice culture

\begin{tabular}{llllllll}
\hline & \multicolumn{3}{c}{ Team number } & & & & \\
\cline { 2 - 7 } & $1(n=13)$ & $2(n=14)$ & $3(n=4)$ & $4(n=25)$ & $5(n=11)$ & $6(n=15)$ & $\begin{array}{c}\text { All respondents } \\
(n=82)\end{array}$ \\
\hline Innovation seeking (m=4) & 4.96 & 5.39 & 4.53 & 4.72 & 4.14 & 4.52 & 4.75 \\
Planning (m=3) & 4.64 & 5.24 & 4.36 & 4.76 & 4.72 & 4.60 & 4.77 \\
Control and loyalty (m=6) & 4.21 & 4.73 & 4.30 & 4.75 & 5.19 & 3.29 & 4.43 \\
Cooperation (m=5) & 5.26 & 5.60 & 5.12 & 5.01 & 5.00 & 5.26 & 5.20 \\
Communication and information (m=5) & 4.86 & 4.83 & 4.02 & 4.42 & 4.48 & 4.21 & 4.51 \\
Valuing staff (m=4) & 5.04 & 5.07 & 4.65 & 4.41 & 4.12 & 4.45 & 4.60 \\
\hline
\end{tabular}

$\mathrm{n}=$ Number of respondents in each team; $\mathrm{m}=$ Number of statements assessed.

Each statement was scored on a seven point scale for importance in the practice, from $7=$ to a great extent to $1=$ not at all 
The team at this point discovered that one of the general practitioners had applied for a grant to introduce a patient complaints procedure which, he claimed, would rectify any aspects raised by the patient satisfaction survey to be conducted between sessions 3 and 4 .

Quality improvement project

Although some topics for quality improvement had been identified, it was decided in session 5 to reject the setting up of a quality improvement group and to omit sessions 6 and 7 .

\section{Reflection}

The lack of commitment to listening to the opinions of other team members, or the facilitator, by one powerful person, meant that the programme could not achieve its aims. However, the evaluation questionnaire showed that the participants had enjoyed the programme and thought that they had benefited in learning how to identify their problems even if they had not yet overcome any of them.

\section{Years later}

The programme was remembered only vaguely, after several prompts by the interviewer. It was reported that improvements had been made and continue to be made, although audits were not undertaken.

TEAM 4

Obstacles

This team was enthusiastic and cooperative throughout the programme. The opportunities in the programme for communication between team members were obvious when the results of the culture questionnaire showed that the doctors held very different views of the practice from other staff. In discussion the doctors seemed to discount the other views as being unrepresentative and unimportant. One team member eventually said that they had real differences of opinion, not merely differences of interpretation of the questions, and that these differences mattered, were an obstacle, and should be put right. This was accepted.

\section{Quality improvement project}

It was agreed that the topic for the first quality improvement group to consider should be improving internal communication and sharing opinions and information. This was a large team (over 30 members had attended session 1) and that was part of the problem. A quality improvement group was appointed which chose to meet without the facilitator being present and decided to collect data to identify the causes of their problems. At a practice meeting between sessions of the programme the quality improvement group had given feedback on the data collected and it had been agreed to set up an internal newsletter and better internal message facilities.

\section{Reflection}

The team decided to omit sessions 6 and 7 of the programme as they thought that they had rectified the immediate problems and their quality agenda was going to take more time than the programme allowed.
3 Years later

A new practice manager had been appointed who reported that the staff still talked about the survival exercise in session 2. It had not been enjoyed but was thought to have been necessary. Since the programme there were quarterly staff meetings and the internal message facilities were still in use. The practice was about to embark with enthusiasm on the local blueprint programme ${ }^{20}$ which the manager hoped would restore their quality activities.

TEAM 5

Obstacles

The members of this team were sceptical at first and anxious about the time commitment required. No team discussions took place between sessions. The first two sessions started late because team members arrived late. It was during feedback after the survival exercise in session 2 that one member declared that she saw quality improvement as disrupting the "natural" hierarchy within the practice. She was against tampering with this system as she thought it would only create unhappiness. Her group's failure to reach a consensus decision reflected her "wrecking power". Discussion of the results of the culture questionnaire identified problems in their working relations. In brainstorming strengths and weaknesses the team could initially identify only weaknesses. The facilitator drew their attention to this and led the discussion into why this negative attitude was so powerful. The team were surprised by how many strengths they could actually identify. Eventually, this team shared ideas and listened to each other and seemed to be open with comments and suggestions, including the earlier sceptics.

\section{Quality improvement project}

A quality improvement group used cause and effect diagrams to identify the data needed to clarify the causes of problems with incoming telephone calls. The data collection showed that each doctor used a different procedure for telephone calls and the receptionists had to adapt to each individual method. A common procedure was agreed by the whole team. This showed consensus decision making in that not all the doctors would have chosen the common procedure for themselves, but thought that their views had been heard and each agreed to support the procedure chosen.

\section{Years later}

The senior partner and practice manager had changed. The practice was about to start the local blueprint programme. ${ }^{20}$ There had been no quality activities since the programme ended that the new practice manager was aware of, although the telephone procedure was still in use.

TEAM 6

Obstacles

This team used the early sessions to air perceived problems with workload, demands of patients, and lack of time to do audit. The results of the culture questionnaire showed 
wide variations in opinion about the team. There was much enthusiasm but no group goals and little coordination to harness the contributions from team members. The team said that they thought that the time was ripe for this programme. However, many of the staff had not been informed of the rescheduled date of session 4, and so although all the doctors were present, no nurses and only half the administrative staff were present. The topic of repeat prescribing was chosen and membership of the quality improvement group were agreed at this session.

\section{Quality improvement project}

The group met with the facilitator and used a flow chart to identify problems in many aspects of the repeat prescription system. The facilitator urged the group to tackle only one or two aspects of the system and collect simple data for a short time. However, when this group presented their findings to the whole team, some members had independently collected detailed data about more aspects of the system than had initially been agreed. The presentation was almost overwhelming and left the team unable to make decisions at that stage on what to do next.

\section{Reflection}

The lack of active cooperation from some of the doctors and the overenthusiasm for data collection by some members of the quality improvement group left the other team members discouraged. The facilitator was unable to persuade the team to deal with only one aspect of the repeat prescription process at a time and the team opted not to complete the programme beyond session 5 . The obstacles had not been overcome, and sabotage from some members helped ensure that they were not likely to be overcome.

\section{Years later}

The survival exercise was remembered but not the quality improvement project. No more quality meetings had been held in the practice. The team functioning was better in that all staff felt able to contribute to discussions. They were just starting the local blueprint programme. $^{20}$

\section{EVALUATION OF THE PROGRAMME BY}

QUESTIONNAIRE

The questionnaire was issued to all 85 team members who attended the programme and 79 $(93 \%)$ participants responded. The mean score for appropriateness of the programme content over all responses was 3.51 (95\% confidence interval ( $95 \% \mathrm{CI}) 3.33$ to 3.69 ) indicating a positive rating. Other scores indicated that the helpfulness, interest, and enthusiasm of the facilitator, and the pace and clarity of presentations and handouts were satisfactory. One respondent commented that it had helped them to talk about problems, which had never been possible before. Another wrote that the communication problems were very serious but she thought that they had at least been acknowledged. There were constructive suggestions on improving the programme-for example, with a simpler culture questionnaire in session one.

\section{Discussion}

This observational study has shown that the initial introduction, with external facilitation, of a selection of the basic quality improvement methods, beyond clinical audit, can be achieved in selected primary healthcare teams. In some teams both the changes introduced in such a programme and interest in quality improvement processes can be sustained over 3 years. It can be feasible, acceptable, effective, and long lasting, but not for every team.

Not all teams, although willing, were ready to embark on the implementation of a quality management programme, with this method of facilitation and support with a preplanned programme. Practices need to consider carefully whether they are prepared to give the commitment required for this process. The teams in this preliminary study took a few small steps towards the introduction of a system to manage quality improvement, but the effort required to run this programme is high for both the participants and the facilitator.

All teams had at least some experience of clinical audit, but none had previously encountered alternative methods for quality improvement. In this programme cause and effect diagrams, flow charts, and brainstorming were all used with data collection not only to locate the problem but also to choose the most effective and acceptable way to improve the situation. The teams also successfully used consensus decision making in selecting the topic to be considered and some used it for agreeing changes to be made.

Three of the six teams in this study did not complete the full programme, although five teams completed a quality improvement group project. Only six teams, out of the 147 invited, actually started the programme. This suggests that commitment to a quality programme is recognised as being demanding but the rewards are not sufficiently attractive. In another study, ${ }^{13}$ even providing up to $£ 2500$ costs in a quality improvement programme did not attract more than 18 out of 360 practices invited.

Although these findings can indicate possible relations between the characteristics of primary healthcare teams and effective quality management, further research is needed to enable measures of characteristics to be used both as predictors of success and to identify development needs of teams.

The study teams, and perhaps many others in primary health care, have a hierarchical structure with the doctors at the summit, responsible for most of the decision making. Although this model can be effective for some aspects of providing care, it can be an obstacle to collaboration by all team members in sharing responsibilities for quality improvement. This can be the case not only for the doctors who find it difficult to accept a flatter power structure and devolve responsibilities, 
but also for some employees who find it difficult to accept more individual responsibility without direction from above. The survival exercise in session two showed that consensus decision making can be very different from the methods previously used for reaching decisions. However, the achievement by team 5 of a consensus decision on a common telephone procedure for all doctors, showed that the method can be learned and used effectively. Unless recognised and put right, resistance from one member to changing the methods of team working can influence the whole group and hinder change. The programme allowed teams the opportunity to learn to deal with obstacles during facilitated discussions but not all teams chose to take that opportunity.

The experiences of this study reinforce the tenet of total quality management that commitment from the top is vital. ${ }^{2}$ The general practitioners should give sufficient leadership to the team so that participation will be thought to be worthwhile. In those teams in which the doctors were not committed, the whole process was difficult. In teams $2,3,4$, and 6 the doctors were the source of difficulties, only some of which were overcome during the programme.

The facilitated programme acted as a catalyst for some teams in starting to develop their quality improvement activities. The facilitators had previous expertise in clinical audit, and they soon were able to adapt those skills to the contents of this programme. The teams rated the content as appropriate for them and, although satisfied with the quality of presentation, indicated where improvements could be made. The interest in quality was still present in some teams after 3 years despite no further facilitation from this study. This was shown by the uptake of the Health Authority blueprint programme ${ }^{20}$ by four practices. However, this also indicates that practices continued to seek expert, external facilitation, and resources to support them in their quality improvement activities.

A national report on quality in primary health care $^{1}$ stated that resources, education, and commitment are needed to introduce quality improvement into primary health care. This programme can provide education and support, but the commitment and time must come from the team, led by the senior members. Thus, it is possible, although not necessarily easy, to implement quality improvement in primary healthcare teams, with a short facilitated programme. Follow up may also enable teams to maintain their quality improvement programmes. This is a role which primary care audit groups are ideally placed to fulfil. However, there is a need for further research to identify the desirable characteristics, skills, and attitudes of primary healthcare teams for the successful introduction of quality improvement programmes, before investment is made in such programmes for large numbers of primary health care teams.

The study provides information on how quality improvement programmes might be developed for primary care teams and general practices.
- Of prime importance is commitment from the doctors in considering any quality issue identified as important by either patients or other team members.

- The team needs to be able to meet together in protected time so that understanding of each others' views on quality can develop.

- External facilitation can help in rectifying problem relations between doctors and others.

- External facilitation acts as a stimulus to maintaining quality activities until they are an accepted team responsibility.

The invaluable contribution of the liaison officers Roz Sorrie and Louise Darling of the then Leicestershire Medical Audit Advisory Group, is warmly acknowledged. We thank all members of the teams who took part. The study was funded by the Trent Regional Health Authority. The foundations for a quality improvement programme was modified considerably in the light of the experiences of presenters and the comments made by participants. A guide to the programme has been written by the research team and liaison officers and published by the Leicestershire Primary Care Audit Group. ${ }^{26}$

1 Clinical Outcomes Group. Clinical audit in primary health care. Report of the primary health care clinical audit working group. London: Department of Health, 1994

2 Oakland J. Total quality management, 2nd edition. Oxford. Butterworth-Heinemann, 1993.

3 Berwick DM. Continuous Improvement as an ideal in health care. N Engl f Med 1989;320:53-6.

4 Berwick DM, Enthoven A, Bunker JP. Quality management in the NHS: the doctor's role: I. BMF 1992;304:235-9.

5 Kaluzny AD, McLaughlin CP, Simpson K. Applying total quality management concepts to public health organizations. Public Health Rep 1992;107:257-64.

6 Martin L. Total quality management in human service organizations. London. Sage Publications, 1993. (Sage Human Services Guide 67.)

7 West MA, Wallace M. Innovation in health care teams. European fournal of Social Psychology 1991;21:303-15.

8 Senior B. Team roles and team performance: Is there really a link?, Fournal of Occupational and Organisational Psychology 1997;70:241-58.

9 Baker R, Thompson J. Innovation in general practice: is the gap between training and non-training practices getting wider?, Br f Gen Pract 1995;45:297-300.

10 Hardy C. Understanding organisations, 4 th edition. Middlesex: Penguin Books, 1993.

11 Walker T. Creating total quality improvement that lasts. National Productivity Review 1992;11:473-8.

12 Fenwick AC. Five easy lessons: a primer for starting a total quality management program. Quality Progress 1991;24: 63-6.

13 Pollitt C. Business approaches to quality improvement: why they are hard for the NHS to swallow. Quality in Health Care 1996;5:104-10.

14 Lawrence M, Packwood T. Adapting total quality management for general practice: evaluation of a programme. Quality in Health Care 1996;5:151-8.

15 Hearnshaw HM, Baker R, Robertson N. Multidisciplinary audit in primary healthcare teams: facilitation by audit support staff. Quality in Health Care 1994;3:164-8.

16 McCowan C, Neville RG, Crombie IK, et al. The facilitator effect: results from a four year follow up of children with asthma. Br f Gen Pract 1997;47:156-60.

17 Dickens P. Quality and excellence in human services. Chichester: Wiley, 1994.

18 Stones E. Quality teaching: a sample of cases. London: Routledge, 1992.

19 Furnham A, Gunter B. Corporate culture: auditing a company's personality. London: Routledge, 1993.

20 Health for Leicestershire. General practice blueprint. An organisational framework for general practice. Leicester: Leicestershire Health, 1997.

21 Kings Fund Organisational Audit. Primary health care organisational standards and criteria. London: King's Fund, 1994.

22 Department of Trade and Industry Enterprise Initiative. Total quality management: the right track [video]. London: DTI, 1990.

23 Human synergistics. Verax survival exercise. Verax House, 93 Fleet Road, Fleet, Hampshire, GU13 8PJ, UK: Verax, 1988

24 Baker R, Hearnshaw HM. Dialogue. Patient satisfaction survey. Eli Lilly National Clinical Audit Centre protocol PS1. Leicester: University of Leicester, 1993.

25 Jarman B. Identification of underprivileged areas. BMF 1983;286:1705-9.

26 Reddish S, Sorrie R, Darling L, et al. Foundations for quality improvement: a facilitator's programme guide. Leicester: Leicestershire Medical Audit Advisory Group, 1995. 